\title{
The Efficacy of Transverse Fixation and Early Exercise in the Treatment of Fourth Metacarpal Bone Fractures
}

\author{
Suk-Ho Moon, Hak-Soo Kim, Sung-No Jung, Ho Kwon \\ Department of Plastic Surgery, College of Medicine, The Catholic University of Korea, Seoul, Korea
}

Background Several techniques have been designed to treat fifth metacarpal fractures reported to be effective. However, these methods cannot be easily applied to the fourth metacarpal due to its central anatomical position. In this study, we sought to analyze the functional outcomes of patients who underwent transverse pinning for a fourth metacarpal bone fracture.

Methods A total of 21 patients were selected and their charts were retrospectively reviewed. After fracture reduction, two transverse Kirchner wires were first inserted from the fifth metacarpal to the third metacarpal transversely at the distal part of the fractured bone, and then another two wires were inserted at the proximal part of the fractured bone. The splint was removed approximately one week postoperatively and the Kirchner wires were removed four to five weeks postoperatively. Patients started active and passive exercise one week after the operation. Pain visual analog scores, total active and passive motion, and the active and passive range of motion of the metacarpophalangeal joint and grip strength were evaluated.

Results Dorsal angulation improved from a preoperative value of $44.2^{\circ}$ to a postoperative value of $5.9^{\circ}$. Six weeks after surgery, functional recovery parameters, such as range of motion and grip strength, had improved to $98 \%$ of the function of the normal side. No major complication was observed.

Conclusions We suggest that the transverse pinning of fourth metacarpal bone fractures is an effective treatment option that is less invasive than other procedures, easy to perform, requires no secondary surgery, minimizes joint and soft tissue injury, and allows early mobilization.

Keywords Metacarpal bones / Bone wire / Fracture fixation
Correspondence: Ho Kwon Department of Plastic Surgery, College of Medicine, The Catholic University of Korea, 271 Cheonbo-ro, Uijeongbu 11765, Korea

Tel: +82-31-820-1114

Fax: +82-31-847-0301

E-mail: kwonho@catholic.ac.kr

This article was presented as a poster at the International Congress of the Korean Society of Plastic and Reconstructive Surgeons on October, 2014 in Seoul, Korea.

No potential conflict of interest relevant to this article was reported.

\section{INTRODUCTION}

Metacarpal bone fractures account for roughly $36 \%$ of all hand fractures, and a quarter of metacarpal fractures occur in the fifth metacarpal bone. Fourth metacarpal bone fractures are less common, and the majority of these fractures are accompanied by fractures in other metacarpal bones, including the fifth metacar$\operatorname{pal}[1]$.

Surgical methods to treat metacarpal bone fractures were first described in 1928 by Lambotte [2], and various surgical methods have since been reported, such as screw and plate fixation, external fixation, intramedullary Kirschner wires (K-wires), tran- 
sverse K-wires, cerclage, and intra-osseous wiring. However, many of these methods have been associated with a range of complications [3-8].

Hands play a vital role in people's everyday lives, and it is important to minimize complications and facilitate the healing process following surgery in order to allow the patients to return to their work and daily activities. Complications usually result from intraoperative joint injuries and prolonged immobilization. Many techniques, including the Bouquet method, have been designed to overcome these shortcomings and have proven to be effective. However, these methods cannot be easily applied to the fourth metacarpal bone due to its central anatomical position.

To date, few reports have assessed the results of transverse pinning of fourth metacarpal bone fractures. We sought to analyze the functional outcomes of patients who underwent transverse fixation for a fourth metacarpal bone fracture.

\section{METHODS}

This study included patients who underwent transverse fixation for a fourth metacarpal bone fracture between October 2010 and September 2013. A total of 21 patients were selected as subjects, excluding cases of hand fractures that occurred adjacent to the fourth metacarpal bone, cases of soft tissue trauma, open fractures, patients with abnormal hand function prior to the surgery, cases in which pin removal was not performed by our department because the patient was lost to follow-up, and cases where the patient's consent was not obtained in person. The following parameters were used as indications for surgery: malrotation was observed after closed reduction, the angulation was more than $30^{\circ}$, and bony contact was less than $70 \%$. Surgery was performed after the hand swelling was reduced.

Surgery was performed under general or regional axillary block anesthesia by using $\mathrm{C}$-arm assistance. The surgeon was seated at the side of the patient's head so that the dorsal and ulnar borders of the patient's hand were close to the surgeon. Reduction was performed using the Jahss maneuver, in which upward pressure is applied to the proximal phalanx with counterpressure applied along the dorsal aspect of the metacarpal bone. If the C-arm showed fracture reduction, a $0.9-\mathrm{mm} \mathrm{K}$-wire insertion was made in the distal region of the fracture segment, perpendicular to the ulnar border of the fifth metacarpal bone, parallel to the hand dorsum, and through the reduced fourth metacarpal bone. If the $\mathrm{C}$-arm showed the fracture segment to be fixed under reduction, a deeper insertion was made through the third metacarpal bone. If it was confirmed to be fixed under reduction, an additional $0.9-\mathrm{mm}$ wire was inserted in the distal region of the frac- ture segment from the fifth metacarpal bone to the third metacarpal bone using the same technique. Later, two additional Kwires were inserted from the fifth to third metacarpal bones in the proximal fracture segment while maintaining the reduction of the fracture using the same method. When the resistance created during pin insertion as the bone cortex was penetrated could be felt, the $\mathrm{C}$-arm was frequently checked to see if contact was being made with the cortex. If no resistance was felt during pin insertion, the pin was pulled back to the ulnar border of the fifth metacarpal bone and insertion was performed from the beginning. Lastly, if the $\mathrm{C}$-arm showed fixation without displacement, passive exercises with continuous fluoroscopy were used to screen for scissoring deformity and displacement of the fixated fracture segment. The K-wires were cut and bent above the skin, and attached to the skin (Fig. 1). After surgery, a protective short arm splint was applied in a protective position. The splint was removed one week postoperatively after the swelling was reduced, and the patients were asked to perform passive exercises, active exercises, and daily activities with only buddy taping on the fourth and fifth digits. However, the patients were instructed to avoid forced active motion or heavy lifting for two months following surgery. The wires were removed when displacement was not seen in X-ray imaging and no pain and tenderness were reported during exercise four weeks after the surgery. Patients with delayed bone union, elderly patients (over 60 years old), and comminuted fracture patients underwent wire removal approximately five weeks postoperatively.

The patients received pin site dressings during their weekly follow-up appointments. On the next day and two, four, six, and ten weeks following the surgery, radiographs were used to check if the fracture segment was maintained, and the patients were asked about their pain using a visual analog scale ranging from one to ten at every follow-up. Two and six weeks after pin removal, the range of motion (ROM) of the injured and uninjured hands was checked with a goniometer. In addition, six weeks following pin removal, the patients were asked to rate their satisfaction with the results of the surgery as poor, fair, good, or excellent, and they were also asked about their subjective recollections of how many days passed after pin removal before they were able to comfortably use their hands. Grip strength was assessed four weeks after pin removal using a dynamometer, and was compared to the opposite, uninjured hand.

\section{RESULTS}

Of the 21 patients, 17 were men and four were women. The mean age was 41.5 years (range, 16-73 years). Twelve individuals (57.1\%) suffered injury to the right hand, while nine (42.9\%) 


\section{Fig. 1. Transverse fixation process seen from the C-arm}

(A) After reduction, a K-wire is inserted into the distal segment of the fracture, (B) and one more K-wire is also inserted into the distal fracture segment. (C) After distal segment fixation, (D) two more K-wires are inserted into the proximal segment, bent and cut above the skin.
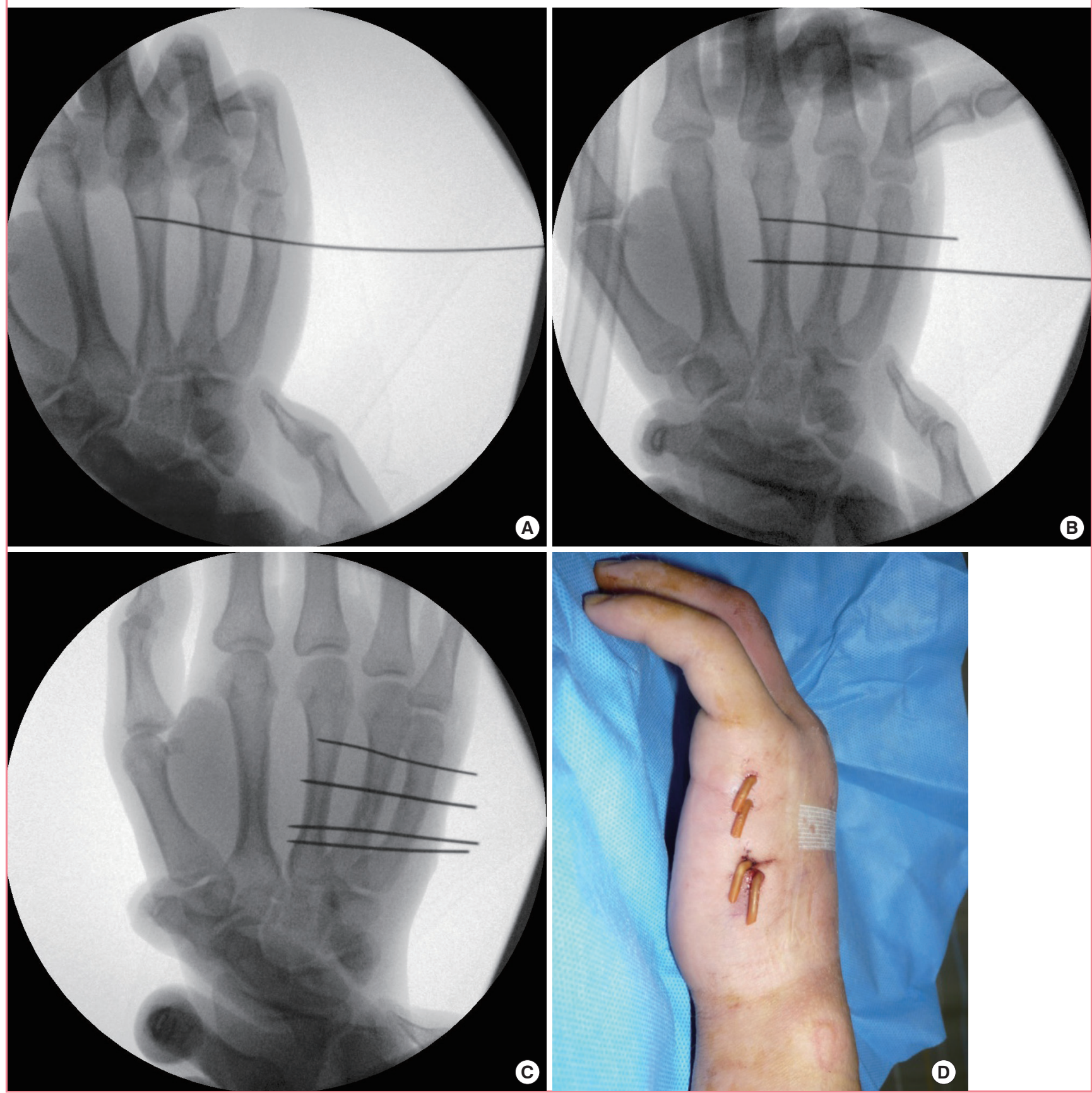

were injured in the left hand. The cause of the injury was striking a person or an object with the fist in eleven patients (52.4\%), blunt trauma in seven patients (33.3\%), and breaking the hand while slipping in three patients (14.3\%). Eight patients (38.1\%) showed transverse fractures, while seven patients $(33.3 \%)$ had spiral fractures and six patients $(28.6 \%)$ had comminuted fractures. The fractures developed predominantly on the shaft (19 patients, $90.5 \%$ ) and occurred much less frequently on the neck (two patients, 9.5\%). The interval from the injury to the surgery was 6.7 days (range, 2-12 days), and the average length of hospitalization was 1.5 days (range, 1-3 days) after surgery. K-wire removal was performed an average of 32.9 days after surgery (range, 28-35 days) (Table 1).

Patients' average pain visual analog scale responses (1-10) were 2.81 (range, $0-4$ ) one day after surgery, 1.61 (range, 0-3) six weeks after surgery, and 0.19 (range, $0-1$ ) ten weeks after 
Table 1. Demographic and clinical characteristics of the patients

\begin{tabular}{|lc|}
\hline Characteristic & No (\%) \\
\hline Age (range, yr) & $41.5(16-73)$ \\
Sex & \\
Male & $17(81)$ \\
Female & $4(19)$ \\
Side & \\
Right & $12(57.1)$ \\
Left & $9(42.9)$ \\
Fracture type & \\
Transverse & $8(38.1)$ \\
Spiral & $7(33.3)$ \\
Comminuted & $6(28.6)$ \\
Fracture location & \\
Neck & $2(90.5)$ \\
Shaft & $19(9.5)$ \\
Base & 0 \\
Injury mechanism & \\
Striking person or objects & $11(52.4)$ \\
Blunt trauma & $7(33.3)$ \\
Slipping down & $3(14.3)$ \\
Total (n) & $21(100)$ \\
Injury to surgery (range, day) & $6.7(2-12)$ \\
Hospitalization (range, day) & $1.5(1-3)$ \\
Fixation to wire removal (range, day) & $32.9(28-35)$ \\
\hline & \\
\hline
\end{tabular}

surgery. The active ROM of the metacarpophalangeal joint (MPJ) was $84.2^{\circ}$ (range, $79^{\circ}-92^{\circ}$ ) six weeks after K-wire removal. The average total active range of motion (TAM) was $255.1^{\circ}$ (range, $234^{\circ}-266^{\circ}$ ). The passive ROM in the injured hand was $94.0^{\circ}$ (range, $88^{\circ}-105^{\circ}$ ) ten weeks after pin removal, and the total passive range of motion (TPM) was $292.4^{\circ}$ (range, $270^{\circ}-315^{\circ}$ ). Two elderly patients (aged 62 and 73 years) initially showed slow recovery of joint motion at the time of K-wire removal, but through physical therapy their hands sufficiently recovered and no discomfort precluded them from using the hand ten weeks postoperatively. Grip strength as tested four weeks following surgery was an average of $33.9 \mathrm{~kg}$ (range, $22-51 \mathrm{~kg}$ ), which was $103.0 \%$ of the value of the uninjured hand (average, $32.9 \mathrm{~kg}$; range, $19-54 \mathrm{~kg}$ ). This outcome was likely observed because the injury occurred in the dominant hand in most cases, which generally has a higher grip strength than the uninjured, nondominant hand. At ten weeks postoperatively, the radiographs showed no cases of re-fracture or displacement in the fracture region. Angulation improved from $44.2^{\circ}$ (range, $30^{\circ}-75^{\circ}$ ) preoperatively to $5.9^{\circ}\left(\right.$ range, $0^{\circ}-12^{\circ}$ ) postoperatively (Table 2).

The patients indicated that it took an average of 13.8 days (range, 5-26 days) to no longer feel discomfort during hand usage after pin removal. Ten patients (47.6\%) indicated that they were highly satisfied with the entire surgery process, six $(23.8 \%)$ indicated that they were somewhat satisfied, five $(23.8 \%)$ indicated that they were somewhat dissatisfied, and none indicated that they
Table 2. Results of the operations and early exercise

\begin{tabular}{|lccc|}
\hline Variable & $\begin{array}{c}\text { Injured } \\
\text { hand }\end{array}$ & $\begin{array}{c}\text { Uninjured } \\
\text { hand }\end{array}$ & $\begin{array}{c}\text { Injured/ } \\
\text { uninjured } \\
(\%)\end{array}$ \\
\hline Active ROM of MPJ $\left(^{\circ}\right)$ & $84.2(79-92)$ & 85.7 & 97.7 \\
TAM $\left(^{\circ}\right)$ & $255.1(234-266)$ & 258 & 98.9 \\
Passive ROM $\left(^{\circ}\right)$ & $94.0(88-105)$ & 95.2 & 98.7 \\
TPM $\left(^{\circ}\right)$ & $292.4(270-315)$ & 297.9 & 98.2 \\
Grip strength (kg) & $33.9(22-51)$ & 32.9 & 103 \\
Angulation $\left(^{\circ}\right)$ & & & \\
$\quad$ Preoperative (range, n) & - & $44.2(30-75)$ & - \\
\hline $\begin{array}{l}\text { ROM, range of motion; MPJ, metacarpophalangeal joint; TAM, total active range } \\
\text { of motion; TPM, total passive range of motion. }\end{array}$ & \\
\hline
\end{tabular}

were highly dissatisfied.

During the outpatient postoperative follow-up period, no pin migration, bending, fracture, or problems were noted, but minor pin site infections were observed in three patients (14.3\%) and successfully treated with oral antibiotics. No occurrences of malrotation, scissoring deformity, or sensory change were observed, but knuckle disappearance was observed in four patients (19.0\%). No other major complications were observed (Table 2).

\section{Case 1}

A 23-year-old male student was injured while hitting a table with his left hand. Fracture and displacement with a $38^{\circ}$ angulation of the fourth metacarpal bone were observed on an X-ray. Seven days after the injury, once the swelling had subsided, surgery was performed as described above. After the surgery, no angulation was observed on an X-ray. On the sixth day after surgery, exercise was initiated after removing the splint. The wires were removed 28 days after surgery, and only self-exercise was conducted without additional physical therapy. Ten weeks after surgery, the active and passive ROM of the MPJ in the injured hand were $87^{\circ}$ and $101^{\circ}$, respectively, and the TAM and TPM were $248^{\circ}$ and $290^{\circ}$, respectively. Using a dynamometer, grip strength was measured as $37 \mathrm{~kg}$ on the injured hand, compared to $41 \mathrm{~kg}$ on the right, uninjured hand. He did not feel discomfort using the injured hand in everyday life and at work twelve days after the removal of the wires. No complications such as malrotation, knuckle disappearance, fracture displacement, were observed during the follow-up period (Fig. 2).

\section{Case 2}

A 32-year-old male patient, who was a professional car dealer, was injured while engaging in water sports through forceful hyperextension of the left hand. Fracture and displacement with $26^{\circ}$ angulation of the fourth metacarpal bone were observed on an X-ray. Fracture of the second and third metacarpal base with carpometacarpal joint dislocation was also observed. Five days 


\section{Fig. 2. Patient with an isolated fourth metacarpal bone fracture}

An isolated left fourth metacarpal bone shaft fracture in a 24-year-old man was treated with closed reduction and transverse K-wire pinning. (A) A preoperative radiograph. (B) A one-week postoperative radiograph. (C) A ten-week postoperative radiograph. (D) Six-weeks after surgery (two weeks after wire removal), the patient was able to flex his fingers freely without discomfort.
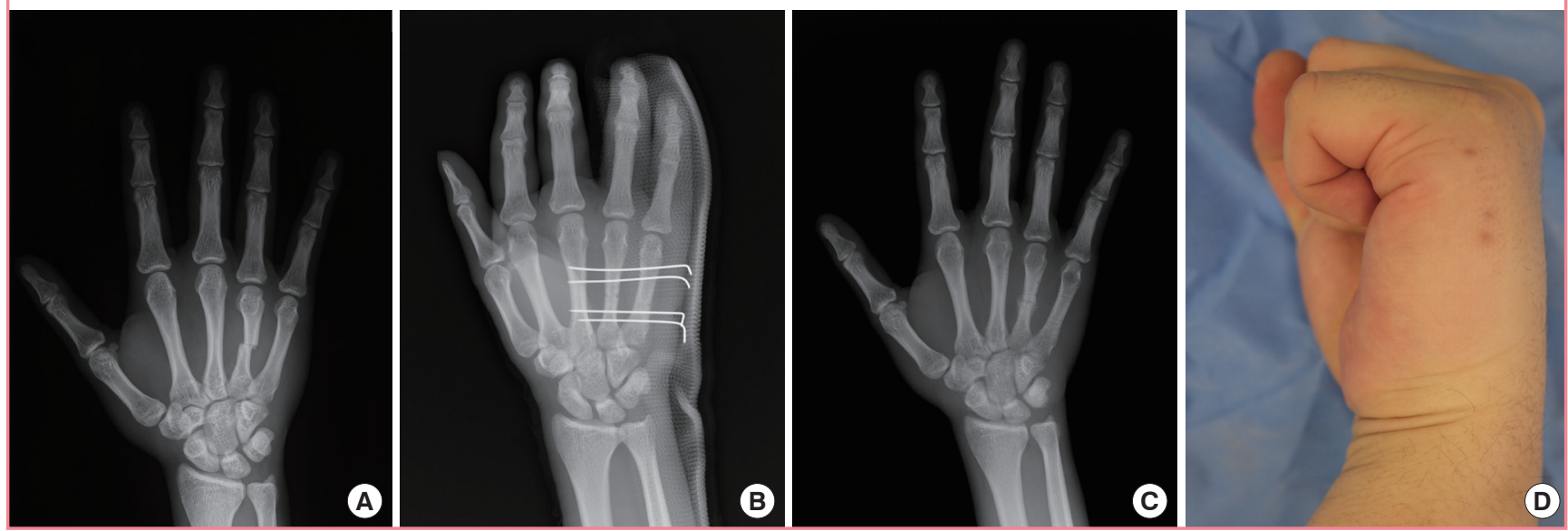

\section{Fig. 3. Second, third, and fourth metacarpal combined fracture}

A 32-year old man with a left fourth metacarpal bone shaft fracture combined with second and third carpometacarpal joint volar dislocation was treated with closure reduction and K-wire pinning. (A, B) Preoperative radiographs. (C) A one-week postoperative radiograph. (D) A four-week postoperative radiograph (just after wire removal).
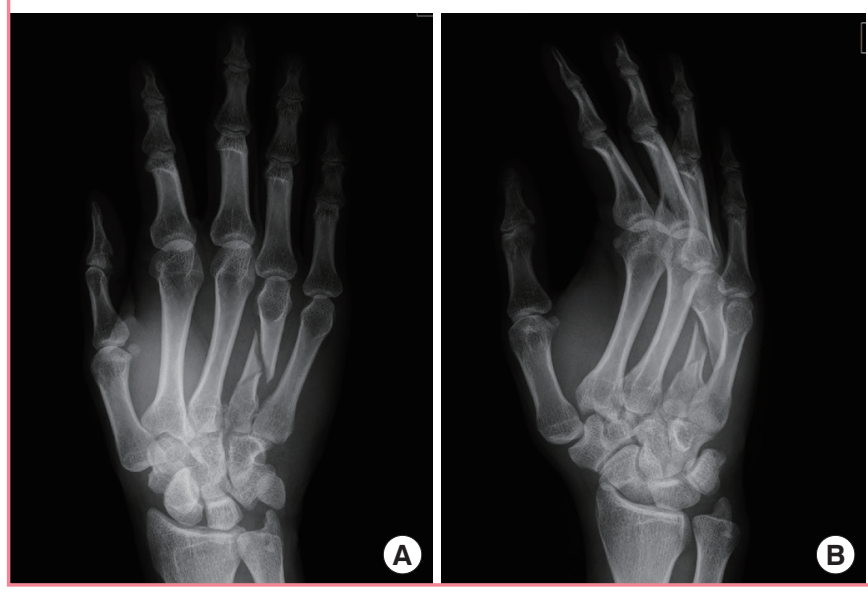

after the injury, closed pinning of the second and third carpometacarpal joints was conducted using K-wires first, in order to support the fourth metacarpal bone fracture area. Reduction and fixation were then performed as described above. After surgery, no angulation was observed on an X-ray. Seven days after surgery, rehabilitation was initiated after removing the splint. The wires were removed 29 days after surgery and self-exercise was conducted without additional physical therapy. Ten weeks after surgery, the active and passive ROM of the MPJ were $89^{\circ}$ and $104^{\circ}$, respectively, and the TAM and TPM were $251^{\circ}$ and $293^{\circ}$, respectively. Using a dynamometer, grip strength was measured as $32 \mathrm{~kg}$ on the injured hand, compared to $34 \mathrm{~kg}$ on the right, uninjured hand. The patient did not feel discomfort using the injured hand in everyday life and at work eight days after the
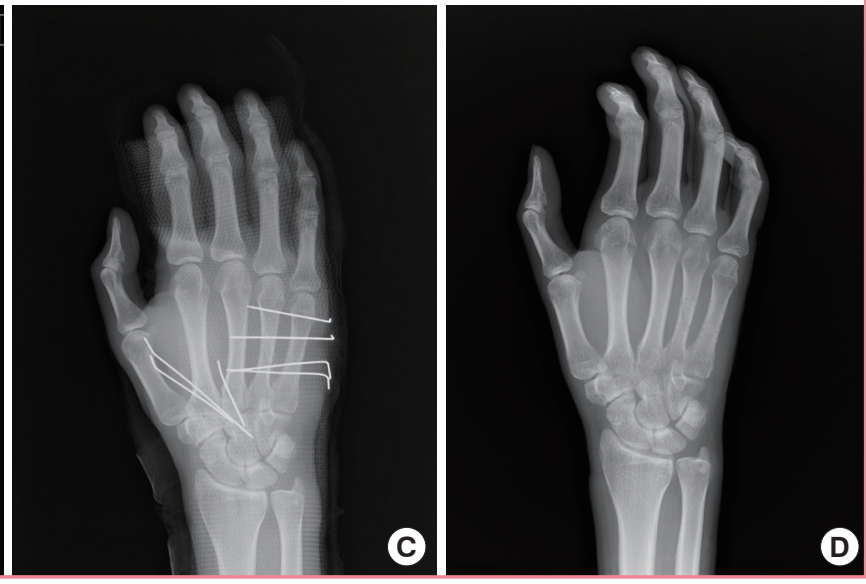

removal of the wires. No complications, including malrotation, knuckle disappearance, and fracture displacement, were observed during the follow-up period. A minimal pin site infection was successfully treated with oral antibiotics (Fig. 3).

\section{DISCUSSION}

Bone fractures of the hand account for roughly $10 \%$ of all fractures in the human body; $46 \%$ of bone fractures are phalangeal bone fractures and another $36 \%$ are metacarpal bone fractures [1]. Hence, metacarpal fractures are frequently encountered by hand surgeons. In many cases they are treatable through conservative management, but in cases where surgical repair is required, various techniques are utilized. Many factors are involved in 
choosing the correct treatment technique, including the location of the fracture (intra- or extra-articular), the fracture pattern (transverse, spiral, oblique, or comminuted), the type of the deformity (angular, rotational, or shortening), open or closed, whether it is accompanied by another bone or soft tissue injury, and fracture stability. In addition, the patient's age, occupation, socioeconomic status, underlying disease, the surgeon's preferences, and the patient's preferences may also play a role. Selecting the proper treatment method is essential to obtaining full and rapid restoration of function [9].

Lambotte [2] first reported the surgical treatment of hand fractures in 1928, and various surgical techniques have been reported since, such as screw and plate fixation, external fixation, intramedullary K-wire, transverse K-wire, cerclage, and intra-osseous wiring [9-18].

Metacarpal plate and screw fixation was commonly performed in the past [10]. This method has the advantage of enabling surgeons to make an incision to confirm the exact location of the fracture, and of using a plate and screws for rigid fixation. Since this method enables exercises to be performed a short time after surgery, the rapid recovery of hand function can be expected. One drawback to this method is that invasive surgery is required to remove the plate and screws. In order to address this shortcoming, several comparison studies with techniques involving bioabsorbable plates and screws have been published [19].

The results of intraosseous cerclage wiring on metacarpal bone fractures have also been described $[15,16]$. Since this technique requires exposure of the fracture area with an incision, the resultant soft tissue damage is greater and scar formation may occur. Moreover, in some cases, wire removal cannot be performed or results in substantial secondary tissue damage when it is performed. External fixation is used for comminuted fractures or when the anatomic skeletal architecture cannot be maintained, but not in simple metacarpal bone fractures $[11,12]$.

Intramedullary nailing has also been reported in antegrade, retrograde, and modified forms [13,20,21]. Intramedullary nailing for little finger metacarpal fractures, which is generally antegrade, inevitably causes injury to the MPJ. It has the drawback of causing fibrosis when joint hemorrhage or tissue injury caused during the procedure heals, which can lead to secondary stiffness or delayed hand function recovery. The Bouquet method was designed to address this shortcoming and has been used in cases of fifth metacarpal fractures. This is an effective method that allows for fixation of the fracture without causing joint injury. However, this method is not suited for the fourth metacarpal bone due to its central anatomical position, and MPJ injury in the fourth metacarpal is unavoidable when intramedullary nailing is used. Moreover, when the fracture is located in a distal re- gion, it becomes increasingly difficult to maintain fixation.

Transverse fixation using K-wires was first reported in 1943, and has mainly been utilized for bone neck fixation of the fifth metacarpal [9]. K-wire is a fixation material familiar to hand surgeons, and has the advantage of assisting reduction by being inserted into the fracture segment and controlled like a joystick. However, if the wire is not sufficiently rigid, it may not be able to firmly hold the fracture area, leading to complications such as pin site infection or pin breakage [22].

Some studies have compared and analyzed intramedullary nailing (the Bouquet method) and the transverse fixation methods for fifth metacarpal bone fractures. Wong et al. [5] compared two groups of patients who underwent transverse fixation or intramedullary K-wiring for fifth metacarpal bone fractures and reported that no differences were found between the two groups. However, Winter et al. [7] reported that intramedullary nailing was superior, but no statistical significance was observed in TAM. MPJ ROM, and grip strength, while a statistically significant improvement was observed in TAM. Sletten et al. [23] stated that no statistically significant functional differences were found between intramedullary and transverse pinning groups; however, the pin site infection rate was higher in the transverse fixation group, and fifth metacarpal bone fractures occurred in three of the 11 patients after pin removal for a fourth metacarpal bone fracture. The authors reasoned that the pin hole in the neighboring metacarpal bone created during the pinning process became a weak point and led to the new fracture. Therefore, the authors concluded that the Bouquet method was preferable.

TAM and TPM are parameters of hand function proposed by the American Society for Surgery of the Hand Clinical Assessment Committee in 1967. TAM of the finger is defined as the sum of active motions of the metacarpophalangeal joint and the two interphalangeal joints of the finger, minus any active extension deficit at the same three joints. TPM also can be checked in the same way during passive extension and flexion. These two tests are useful parameters for assessing digital motion. According to these criteria, a total active flexion score higher than $120^{\circ}$ is considered excellent, $80^{\circ}-120^{\circ}$ is good, and lower than $80^{\circ}$ is considered poor. TAM of the normal hand is also measured, and the function of the hand that underwent operation is rated as excellent when a $100 \%$ match is observed with the normal hand, while a match of $75 \%$ to $95 \%$ is considered good, and a match of below $75 \%$ is considered poor [24,25].

We treated fourth metacarpal bone fractures using transverse fixation. Two pins were inserted on either side of the fracture segment, for a total of four pins. The inserted pins fixed the metacarpals from the third to the fifth metacarpal bones to prevent rotation and angulation that could occur with the pin as the piv- 
ot, allowing them to remain rigid even during early exercise. In some previous studies that used only one wire per fracture segment, it was reported that angulation occurred in some cases, leading to the suggestion that at least two-wire fixation should be used [14]. Our study used two pins for each fracture segment. Using the intact third and fifth metacarpal bones as pillars, it was deemed that using two pins for each segment would allow greater resistance against bending fracture force and rotation. In fact, no displacement or nonunion occurred when the patients began exercising one week postoperatively. We plan to conduct a cadaveric study to investigate how the number of pins affects the amount of force.

No new fractures developed in the neighboring metacarpals in our study. Moreover, only one study on fifth metacarpal fractures has reported newly developed fractures. It is theoretically possible for multiple pin holes from pinning to result in neighboring metacarpal fractures, and such cases have indeed been reported. The chances of this can be reduced by taking care to insert the pin in a different region by adjusting its level in cases where proper fixation does not occur after pin insertion into the metacarpal bone.

Our study has the drawback of being a retrospective study with no control group, as well as having a relatively small sample size. However, considering the scarcity of data on post-surgical functional recovery and patient satisfaction after procedures to treat isolated fourth metacarpal bone fractures, we believe the results of this study to be of value.

\section{REFERENCES}

1. Hove LM. Fractures of the hand. Distribution and relative incidence. Scand J Plast Reconstr Surg Hand Surg 1993;27: 317-9.

2. Lambotte A. Contribution a la chirurgie conservatrice de la main doms les traumatismes. Arch Franco Belges Chir 1928; 31:759-61.

3. Foucher G. "Bouquet" osteosynthesis in metacarpal neck fractures: a series of 66 patients. J Hand Surg Am 1995;20: S86-90.

4. Gonzalez MH, Igram CM, Hall RF Jr. Flexible intramedullary nailing for metacarpal fractures. J Hand Surg Am 1995; 20:382-7.

5. Wong TC, Ip FK, Yeung SH. Comparison between percutaneous transverse fixation and intramedullary K-wires in treating closed fractures of the metacarpal neck of the little finger. J Hand Surg Br 2006;31:61-5.

6. Orbay JL, Touhami A. The treatment of unstable metacarpal and phalangeal shaft fractures with flexible nonlocking and locking intramedullary nails. Hand Clin 2006;22:27986.

7. Winter M, Balaguer T, Bessiere C, et al. Surgical treatment of the boxer's fracture: transverse pinning versus intramedullary pinning. J Hand Surg Eur Vol 2007;32:709-13.

8. Westbrook AP, Davis TR, Armstrong D, et al. The clinical significance of malunion of fractures of the neck and shaft of the little finger metacarpal. J Hand Surg Eur Vol 2008;33: 732-9.

9. Galanakis I, Aligizakis A, Katonis P, et al. Treatment of closed unstable metacarpal fractures using percutaneous transverse fixation with Kirschner wires. J Trauma 2003;55:509-13.

10. Ceim U. Small fragment set manual. In: Heim U, Pfeiffer $\mathrm{KM}$, Oberli $\mathrm{K}$, et al., editors. Internal fixation of small fractures: technique recommended by the AO-ASIF Group. 3rd ed. New York: Springer-Verlag; 1982. p.480-4.

11. Cooney WP III, Smith DK. External fixation of the upper extremity. In: American Academy of Orthopaedic Surgeons, editor. AAOS Instructional Course Lectures. St. Louis: CV Mosby; 1987. p.445-69.

12. Riggs SA Jr, Cooney WP 3rd. External fixation of complex hand and wrist fractures. J Trauma 1983;23:332-6.

13. Faraj AA, Davis TR. Percutaneous intramedullary fixation of metacarpal shaft fractures. J Hand Surg Br 1999;24:76-9.

14. Paul AS, Kurdy N, Kay PR. Fixation of closed metacarpal shaft fractures: transverse K-wires in 22 cases. Acta Orthop Scand 1994;65:427-9.

15. Gropper PT, Bowen V. Cerclage wiring of metacarpal fractures. Clin Orthop Relat Res 1984;(188):203-7.

16. Lister G. Intraosseous wiring of the digital skeleton. J Hand Surg Am 1978;3:427-35.

17. Freeland AE, Orbay JL. Extraarticular hand fractures in adults: a review of new developments. Clin Orthop Relat Res 2006; 445:133-45.

18. Sohn RC, Jahng KH, Curtiss SB, et al. Comparison of metacarpal plating methods. J Hand Surg Am 2008;33:316-21.

19. Waris E, Ashammakhi N, Raatikainen T, et al. Self-reinforced bioabsorbable versus metallic fixation systems for metacarpal and phalangeal fractures: a biomechanical study. J Hand Surg Am 2002;27:902-9.

20. Han SH, Rhee SY, Lee SC, et al. Percutaneous retrograde intramedullary single wire fixation for metacarpal shaft fracture of the little finger. Eur J Orthop Surg Traumatol 2013; 23:883-7.

21. Lee SK, Kim KJ, Choy WS. Modified retrograde percutaneous intramedullary multiple Kirschner wire fixation for treatment of unstable displaced metacarpal neck and shaft fractures. Eur J Orthop Surg Traumatol 2013;23:535-43. 
22. Hong SJ, Lee HJ, Kim JY, et al. K-wire breakage during metalware removal due to a defective K-wire shaft. Arch Plast Surg 2013;40:655-7.

23. Sletten IN, Nordsletten L, Husby T, et al. Isolated, extra-articular neck and shaft fractures of the 4th and 5th metacarpals: a comparison of transverse and bouquet (intra-medullary) pinning in 67 patients. J Hand Surg Eur Vol 2012;37: 387-95.
24. Soni A, Gulati A, Bassi JL, et al. Outcome of closed ipsilateral metacarpal fractures treated with mini fragment plates and screws: a prospective study. J Orthop Traumatol 2012;13: 29-33.

25. Macey AC, Burke FD, Abbott K, et al. Outcomes of hand surgery: British Society for Surgery of the Hand.J Hand Surg Br 1995;20:841-55. 